\title{
Liderazgo femenino en medicina y su evolución en el tiempo: 50 años de análisis bibliométrico
}

\section{Evolution of Female Leadership in Medicine. Bibliometric Analysis and Evaluation of the Last 50 Years}

\author{
1 Uróloga, Uroclin, Medellín, Colombia \\ 2 Uróloga Pediatra, Hospital Infantil Universitario San José, Bogotá, \\ Colombia \\ ${ }^{3}$ Uróloga - Transplante renal, Clínica Foscal, Bucaramanga, Colombia \\ ${ }^{4}$ Uróloga - Clínica Santa Maria del Lago, Bogotá, Colombia \\ ${ }^{5}$ Uróloga - Magister en Epidemiología, Hospital La Samaritana, \\ Bogotá, Colombia \\ ${ }^{6}$ Uróloga - Clínica Sagrado Corazón, Medellín, Colombia
}

Catalina Solano Mendoza ${ }^{1}$ Andrea Patricia Bolaños Gomez ${ }^{2}$ Veronica Tobar ${ }^{3}$ Luisana Castillo Carvajal $^{4}$ Johanna Vega ${ }^{5}$ Luz Marina Bernal Jaimes ${ }^{6}$

Urol Colomb 2021;30(3):e171-e178.

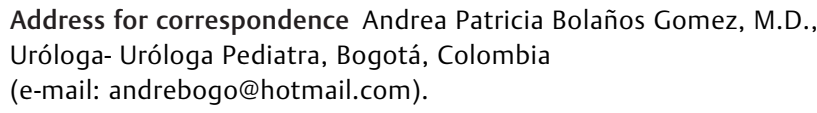

Address for correspondence Andrea Patricia Bolaños Gomez, M.D. Uróloga- Uróloga Pediatra, Bogotá, Colombia (e-mail: andrebogo@hotmail.com).

\section{Resumen}

\section{Palabras claves}

- Liderazgo

- mujer

- trabajo

- médicos mujeres

- educación médica

- inequidad de genero
Objetivo Realizar un análisis bibliométrico sobre el liderazgo femenino en medicina para conocer el panorama actual y dirigir futuras investigaciones.

Métodos Se realizó un análisis bibliométrico descriptivo retrospectivo de la literatura disponible en MEDLINE en relación con el liderazgo de mujeres médicas de 1973 al 2019. Se utilizó Pubmed y FABUMED, y la siguiente estrategia: ("Leadership"[Mesh]) AND "Physicians, Women"[Mesh] (1973:2019[dp]). Se incluyeron artículos originales, de revista, ensayos, informes científicos y reseñas, para analizar toda la literatura indexada relacionada.

Para la obtención del factor de impacto (FI), se utilizó el Journal Citation Reports (7) 2017/2018 de las revistas encontradas para estimar la calidad de cada una de ellas.

Resultados Un total de 310 referencias fueron encontradas en 139 revistas publicadas, el $71,2 \%$ fueron artículos originales, el resto revisiones. Hubo un incremento en las publicaciones de 1996 al 2019 en un 11,9\%. Las revistas con mayor porcentaje de publicaciones fueron: Academic Medicine y J Womens Health (Larchmt), con 7,7\% y 7,1\%. Los países con mayores publicaciones fueron Estados Unidos, con 66 (56\%) y Reino Unido con 10 (8,6\%). América latina tiene cuatro publicaciones y Colombia no cuenta con ninguna publicación indexada.

Conclusión El liderazgo femenino en medicina y las publicaciones sobre el tema han aumentado en las últimas décadas en países industrializados. Por su parte, Latinoamérica debe aumentar sus esfuerzos en publicar en revistas con alto factor de impacto para trabajar por la equidad de género. received

September 16, 2020

accepted

December 22, 2020
DOI https://doi.org/

$10.1055 / \mathrm{s}-0041-1724045$

ISSN 0120-789X.

e ISSN 2027-0119.
(C) 2021. Sociedad Colombiana de Urología. All rights reserved. This is an open access article published by Thieme under the terms of the Creative Commons Attribution-NonDerivative-NonCommercial-License, permitting copying and reproduction so long as the original work is given appropriate credit. Contents may not be used for commercial purposes, or adapted, remixed, transformed or built upon. (https://creativecommons.org/ licenses/by-nc-nd/4.0/)

Thieme Revinter Publicações Ltda., Rua do Matoso 170, Rio de Janeiro, RJ, CEP 20270-135, Brazil 


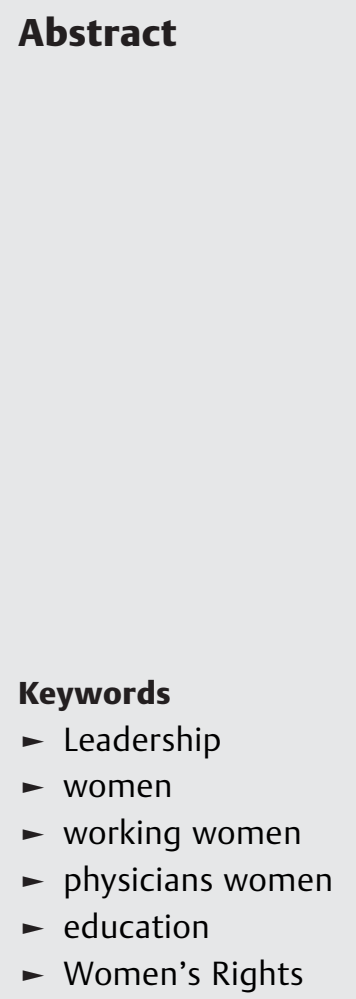

Objective Perform a biometrical analysis of female leadership in medicine, in order to know the actual perspective and guide future research.

Materials and Methods: A retrospective descriptive bibliometric analysis of the literature available in MEDLINE was performed in relation to the leadership of female physicians from 1973 to 2019, through MEDLINE database, using GoPubmed and FABUMED. The following strategy was deployed: ("Leadership"[Mesh]) AND "Physicians, Women"[Mesh] (1973:2019[dp]). Original articles, journal articles, essays, scientific reports and reviews were included to analyze all related indexed literature. To obtain the impact factor (FI), the Journal Citation Reports (7) 2017/2018 of the journals found was used to estimate the quality of each one of them.

Results A total of 310 references were found in 139 published journals,

$71.2 \%$ were original articles; the remaining $22 \%$ were reviews. There was a $11.9 \%$ increase in publications from 1996 to 2019. The journals with the highest rate of publications were: Academic Medicine and J Womens Health (Larchmt) with $7.7 \%$ and $7.1 \%$. Countries with the highest number of publications were United States with 66 (56\%), and United Kingdom with 10 (8.6\%). Latin America has four publications, while Colombia has none.

Conclusion Female leadership in the medical field and the publications regarding this subject have increased in the last decades, mainly in developed countries. Latin America has to make significant efforts to produce more publications in journals with high impact factor, in order to close the gender gap in the medical field.

\section{Introducción}

En las últimas dos décadas se ha logrado un aumento significativo en el ingreso de mujeres a las escuelas de medicina, pasando de un $8 \%$ en 1970 a $42 \%$ en $1997,{ }^{1}$ así como el aumento en el número de mujeres en puestos docentes; sin que eso se pueda considerar un concepto real de paridad de género. El número de mujeres líderes, con cargos de alto rango y puestos directivos es bajo y no es proporcional a la cantidad que ingresa en la educación profesional. ${ }^{2}$

Esa desigualdad puede ser explicada por varios factores; algunos como menor preparación postdoctoral, mayor responsabilidad familiar, diferencias en la asignación de recursos, roles de género tradicionales, manifestaciones de sexismo en el entorno médico y falta de tutorías efectivas. ${ }^{3}$ Múltiples obstáculos están englobados en el fenómeno llamado "techo de cristal", el cual se refiere al grupo de barreras invisibles, que impiden que una mujer con todos los reconocimientos, logros y éxitos, pueda acceder a cargos directivos altos. ${ }^{4}$

La inequidad ha generado la necesidad de crear y fomentar estrategias para el continuo liderazgo, donde se buscan cuatro objetivos principales: proporcionar educación a las mujeres, crear igualdad de oportunidades, aumentar la visibilidad y valorar el trabajo femenino. Esas estrategias son necesarias, aunque no son suficientes para el avance, ya que se requiere de un cambio cultural para tener una verdadera transformación. ${ }^{2,5}$

$\mathrm{Y}$ ya que esos temas son recientes y no se encontró literatura al respecto en nuestro país, este estudio tiene como objetivo realizar un análisis bibliométrico sobre el liderazgo femenino en medicina para conocer el panorama actual y dirigir futuras investigaciones. ${ }^{6}$

\section{Métodos}

Se realizó un análisis bibliométrico descriptivo de corte retrospectivo de la literatura médica disponible en la base de datos de MEDLINE en relación con el liderazgo de mujeres médicas teniendo en cuenta el rango de años disponibles desde 1973 hasta el año 2019.

La base de datos MEDLINE fue consultada en Enero 2020 a través de PubMed y FABUMED, utilizando la estrategia de búsqueda: ("Leadership"[Mesh]) AND "Physicians, Women"[Mesh] (1973:2019[dp]). Se incluyeron artículos originales de investigación, artículos de revista, ensayos, informes científicos y reseñas, con el fin de analizar toda la literatura médica indexada en relación con ese tema.

Se utilizó la herramienta estadística de PubReminer y FABUMED para la obtención de la información de las variables a analizar (número total de publicaciones y revistas, número y porcentaje de publicaciones por revista y país, y patrón de producción científica a lo largo del período estudiado, autores, tipos de documento y artículos más citados). Para la obtención del factor de impacto (FI), (medida bibliométrica utilizada para medir la calidad de las revistas y de los artículos publicados) utilizamos el Journal Citation Reports ${ }^{7}$ 2017/2018 de las revistas encontradas para estimar la calidad de cada una de ellas.

Mediante el software VosViewer que utiliza técnicas de agrupamiento, ${ }^{8,9}$ se exploraron las interacciones de palabras 
clave, palabras clave de título y resumen de la literatura encontrada, con el fin de mapear el dominio del conocimiento científico y revelarle al lector una manera simple y rápida de ver los datos. Se adoptó el análisis de co-ocurrencia para investigar las áreas populares y direcciones de investigación y fue clave para monitorear desarrollos en áreas científicas.

Posterior a ello, analizamos los resultados de forma estadística y descriptiva y con base en esa información, elaboramos tablas y gráficas en Microsoft Office Excel 2017.

\section{Resultados}

Un total de 310 referencias fueron encontradas en 139 revistas publicadas, $221(71,2 \%)$ fueron artículos originales. Durante la mitad del primer periodo (1973-1996) se encontraron $37(11,9 \%)$ publicaciones y en la segunda mitad (1996-2019) 273 (88\%), lo que demuestra un crecimiento en las publicaciones de 7,6 veces (- Figura 1).

En la - Tabla 1 se mostraron las veinte revistas con mayor número de publicaciones durante el periodo de 1973 a 2019, así como el número de artículos publicados en cada una de ellas y el FI para el año 2017/2018 según el Journal Citation Reports. ${ }^{7}$ Las revistas con mayor porcentaje de publicaciones fueron: Academic Medicine con 7,7\% (24), seguida por $J$ Womens Health (Larchmt) con 7,1\%(21) y con igual número de publicaciones en el cuarto y quinto lugar J Am Coll Radiol y Lancet con 2,5\%. - Figura 2

El segundo punto que se desatacó, es el tipo de revistas que publicaron al respecto, donde a pesar del aumento de publicaciones del tema, ellas se han realizado principalmente en revistas científicas con bajo FI. Es así como se encontró, que en el ranking de las veinte revistas con mayor número de publicaciones acerca de liderazgo femenino, los tres primeros puestos no superan un FI de 8, y solo cuatro revistas tienen un FI alto, el mayor de ellos, El New England Journal of Medicine, en el puesto 10 con un FI de 70,67.

De las 310 referencias publicadas en 27 países, Estados Unidos ocupó el primer puesto con 66 (21,2\%), como se ve en la - Figura 3 seguido del Reino Unido con diez (3,2\%), Canadá con seis (1,9\%), Australia con cinco (1,6\%) e India con tres ( $0,9 \%)$. El país de América Latina con mayor número de publicaciones fue México con tres referencias, seguido de Brasil con una $(0,3 \%)$. Colombia no cuenta con publicaciones indexadas.

Las publicaciones acerca del liderazgo en mujeres médicas empezaron hace más de 40 años, sin embargo, el aumento en las publicaciones se da a partir del 2011, con la publicación del manuscrito titulado "Is there still a glass ceiling for women in academic surgery? de la revista Annals of Surgery con FI 10,13 , siendo el más citado y logrando el auge del término "techo de cristal". - Tabla 2

\section{Análisis por mapeo}

Mediante la herramienta VOSviewer se realizó un análisis por agrupamiento de los 310 artículos encontrados. Se mapearon las palabras clave, título y resumen nombradas un mínimo de 5 veces. En total, se distribuyeron en cinco grupos las 220 palabras encontrados ( $\mathbf{- T a b l a ~} \mathbf{3}$ )

Se describieron los tres principales grupos (rojo, verde y azul). El Grupo 1 (rojo) hace referencia a las escuelas / academias: relacionadas con la diversidad de género, inclusión, y liderazgo femenino. El Grupo 2 (verde), proporción de miembros en altos cargos y juntas directivas,

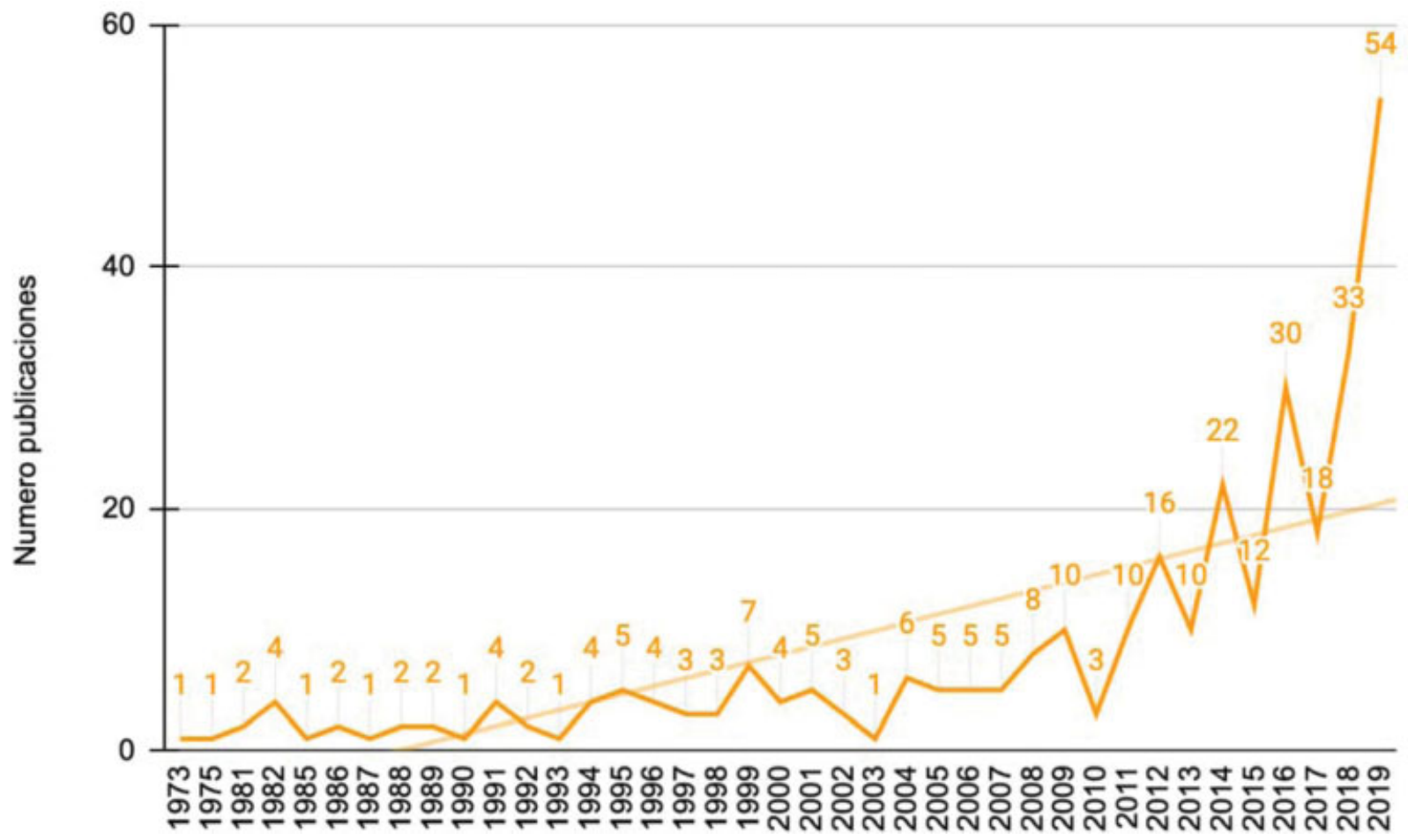

Fig. 1 Distribución de publicaciones por año des 1973 hasta 2019. 
174 Liderazgo femenino en medicina y su evolución en el tiempo Mendoza y col

Tabla 1 Listado de revistas con publicación de artículos sobre liderazgo de mujeres médicas durante el período 1973 a 2019 , su FI para el año 2017-2018, el número de publicaciones, el idioma. (*no se encontró el FI para este periodo de tiempo)

\begin{tabular}{|l|l|l|l|l|}
\hline Revista & Artículos & $\begin{array}{l}\text { Factor de impacto } \\
2017-2018\end{array}$ & Idioma & País \\
\hline J Urol & 20 & 5,6 & Inglés & Estados Unidos \\
\hline Urology & 17 & 1,8 & Inglés & Estados Unidos \\
\hline Urologe A & 6 & $*$ & Alemán & Alemania \\
\hline Prog Urol & 4 & 0,5 & Francés & Francia \\
\hline J Endourol & 3 & 2,2 & Inglés & Estados Unidos \\
\hline J Pediatr Urol & 3 & 1,7 & Inglés & Inglaterra \\
\hline World J Urol & 3 & 2,7 & Inglés & Estados Unidos \\
\hline Fed Pract & 2 & $*$ & $*$ & $*$ \\
\hline Int J Urol & 2 & 2,1 & Inglés & Japón \\
\hline Int Urogynecol J Pelvic Floor Dysfunct & 2 & $*$ & Inglés & Inglatera \\
\hline Investig Clin Urol & 2 & 1,6 & Inglés & Corea del Sur \\
\hline J Am Coll Surg & 2 & 4,4 & Inglés & Estados Unidos \\
\hline Transl Androl Urol & 2 & 2,1 & Inglés & China \\
\hline Urol Int & 2 & 1,4 & Inglés & Suiza \\
\hline Urol J & 2 & 1,4 & Inglés & Irán \\
\hline Actas Urol Esp & 1 & 1,1 & Español & España \\
\hline Andrology & 1 & 3,1 & Inglés & Estados Unidos \\
\hline Ann Surg Oncol & 1 & 3,6 & Inglés & Estados Unidos \\
\hline Arch Intern Med & 1 & 17,3 & Inglés & Estados Unidos \\
\hline Arch Surg & 1 & $*$ & Inglés & Estados Unidos \\
\hline
\end{tabular}

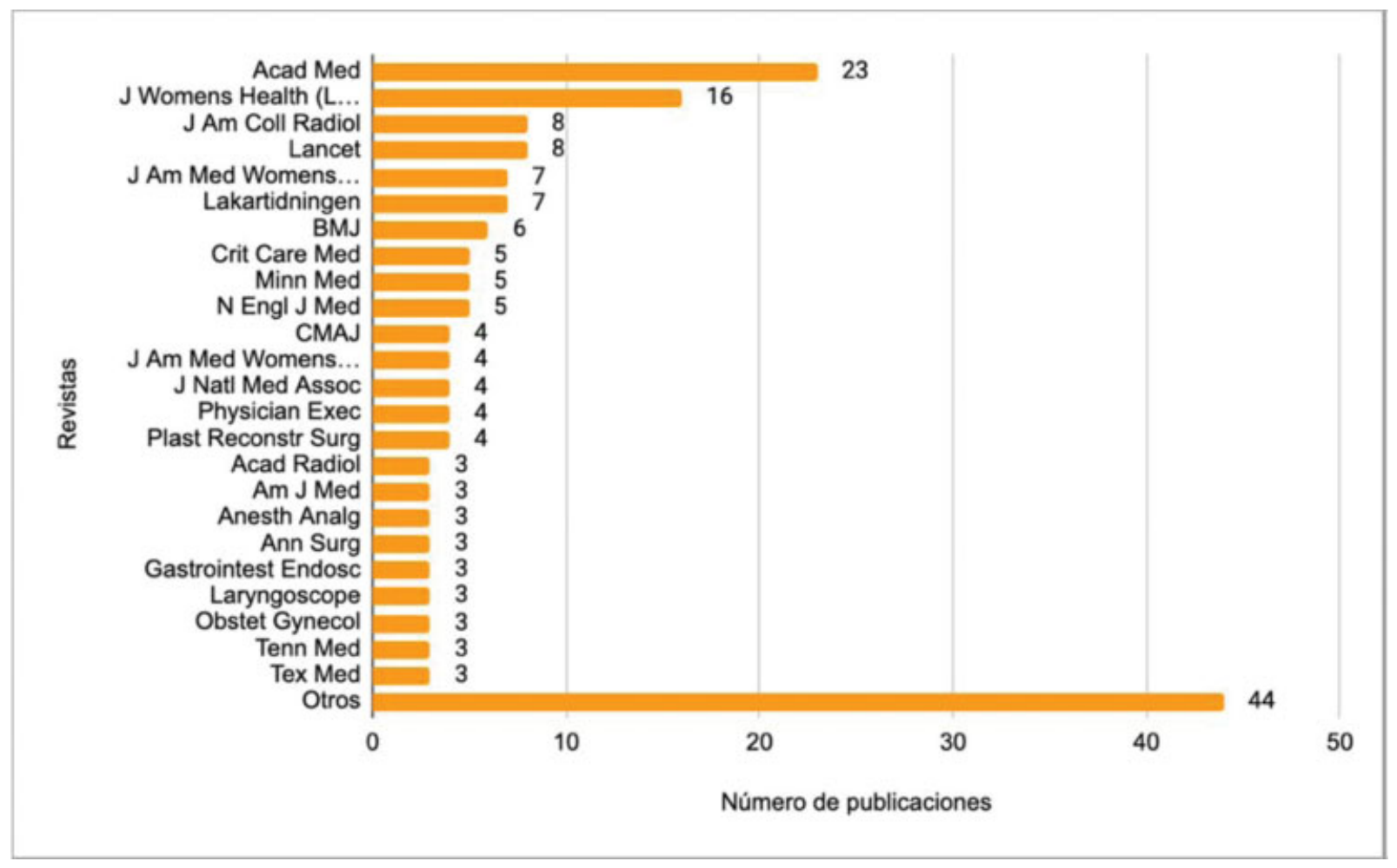

Fig. 2 Distribución porcentual de revistas con publicación sobre liderazgo en mujeres médicas durante el período $1973-2019$. 


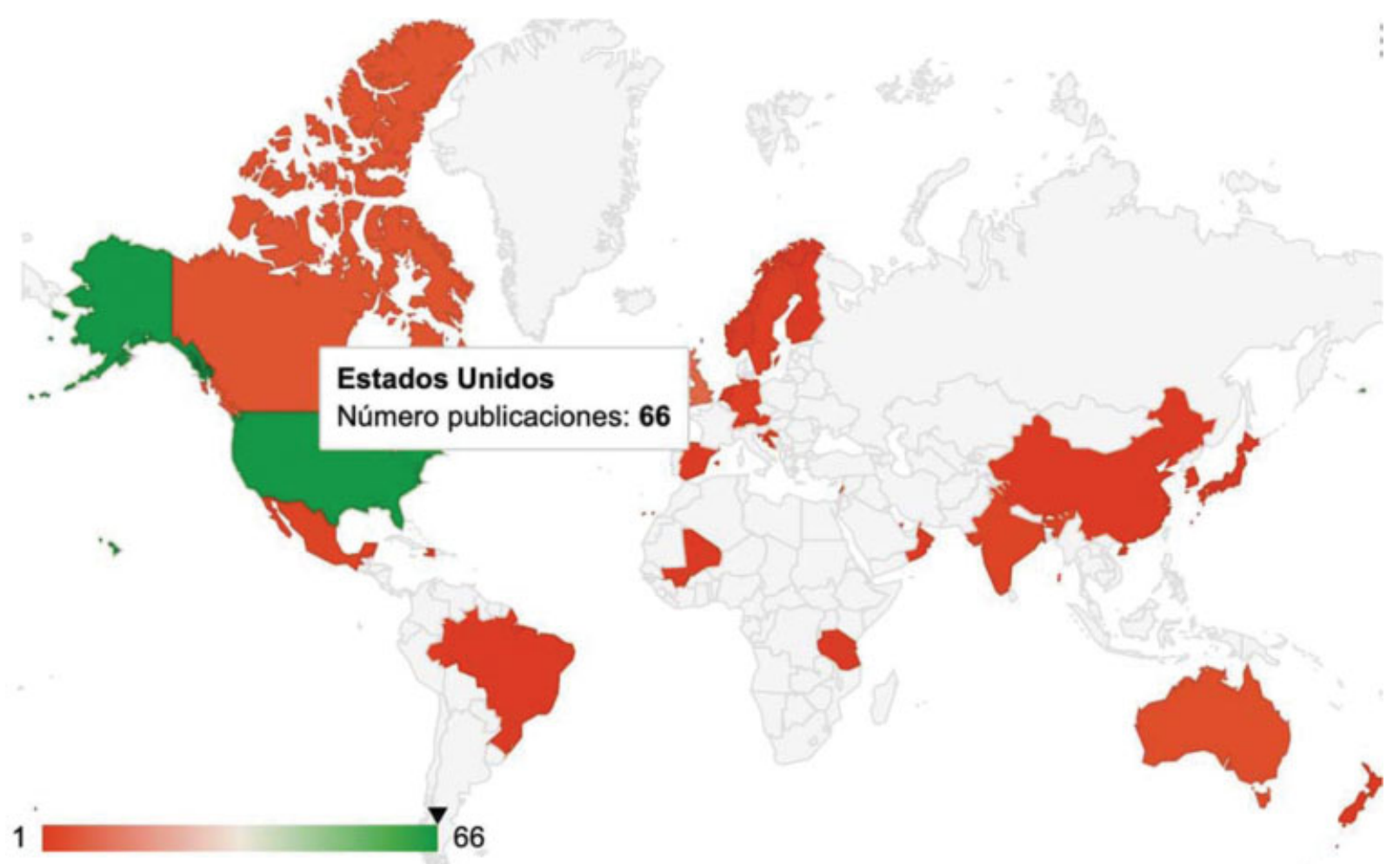

Fig. 3 Países participantes en las publicaciones de liderazgo en mujeres médicas desde 1973-2019.

Tabla 2 Listado de publicaciones sobre liderazgo de mujeres médicas mas citados

\begin{tabular}{|c|c|c|c|c|c|}
\hline Autores & Título & Año & Revista & Citaciones & $\begin{array}{l}\text { Tipo de } \\
\text { documento }\end{array}$ \\
\hline Zhuge Y., y col. & $\begin{array}{l}\text { Is there still a glass ceiling for women in } \\
\text { academic surgery? }\end{array}$ & 2011 & Annals of Surgery & 196 & Artículo \\
\hline Jagsi R., y col. & $\begin{array}{l}\text { Gender differences in the salaries of physician } \\
\text { researchers }\end{array}$ & 2012 & $\begin{array}{l}\text { JAMA - Journal of the American } \\
\text { Medical Association }\end{array}$ & 171 & Artículo \\
\hline Carr P.L., y col. & $\begin{array}{l}\text { Inadequate Progress for Women in Academic } \\
\text { Medicine: Findings from the National Faculty } \\
\text { Study }\end{array}$ & 2015 & Journal of Women's Health & 119 & Artículo \\
\hline Girod S., y col. & $\begin{array}{l}\text { Reducing implicit gender leadership bias in } \\
\text { academic medicine with an educational } \\
\text { intervention }\end{array}$ & 2016 & Academic Medicine & 88 & Artículo \\
\hline Eloy J.A., y col. & $\begin{array}{l}\text { Gender disparities in research productivity } \\
\text { among } 9952 \text { academic physicians }\end{array}$ & 2013 & Laryngoscope & 77 & Artículo \\
\hline Butkus R., y col. & $\begin{array}{l}\text { Achieving gender equity in physician } \\
\text { compensation and career advancement: } \\
\text { A position paper of the American College of } \\
\text { Physicians }\end{array}$ & 2018 & Annals of Internal Medicine & 68 & Artículo \\
\hline Abelson J.S., y col. & $\begin{array}{l}\text { The climb to break the glass ceiling in } \\
\text { surgery: trends in women progressing from } \\
\text { medical school to surgical training and } \\
\text { academic leadership from } 1994 \text { to } 2015\end{array}$ & 2016 & American Journal of Surgery & 61 & Artículo \\
\hline Silver J.K., y col. & $\begin{array}{l}\text { Where Are the Women? The Underrepresen- } \\
\text { tation of Women Physicians Among Recog- } \\
\text { nition Award Recipients From Medical } \\
\text { Specialty Societies }\end{array}$ & 2017 & $\mathrm{PM}$ and $\mathrm{R}$ & 54 & Artículo \\
\hline Rohde R.S., y col. & $\begin{array}{l}\text { Where Are the Women in Orthopaedic } \\
\text { Surgery? }\end{array}$ & 2016 & $\begin{array}{l}\text { Clinical Orthopaedics and Relat- } \\
\text { ed Research }\end{array}$ & 47 & Artículo \\
\hline Rochon P.A., y col. & $\begin{array}{l}\text { Women in academic medicine leadership: } \\
\text { Has anything changed in } 25 \text { years' }\end{array}$ & 2016 & Academic Medicine & 47 & Artículo \\
\hline
\end{tabular}


Tabla 3 Palabras clave con mayor número de co-ocurrencia en titulo y resumen

\begin{tabular}{|l|l|l|}
\hline Término & Ocurrencia & Relevancia \\
\hline school & 55 & 0,08 \\
\hline member & 46 & 0,20 \\
\hline proportion & 45 & 0,50 \\
\hline difference & 39 & 0,51 \\
\hline success & 34 & 0,39 \\
\hline chair & 29 & 0,24 \\
\hline diversity & 28 & 0,71 \\
\hline health & 26 & 0,89 \\
\hline increase & 25 & 0,50 \\
\hline gender equity & 24 & 0,90 \\
\hline resident & 24 & 0,74 \\
\hline interview & 23 & 1,05 \\
\hline culture & 23 & 0,81 \\
\hline academin rank & 22 & 0,93 \\
\hline approach & 22 & 0,90 \\
\hline theme & 21 & 1,32 \\
\hline rank & 21 & 0,53 \\
\hline full professor & 21 & 0,47 \\
\hline work life balance & 20 & 1,00 \\
\hline support & 20 & 0,83 \\
\hline women physician & 20 & 0,71 \\
\hline medical student & 20 & 0,48 \\
\hline evidence & 19 & 0,86 \\
\hline & & \\
\hline
\end{tabular}

tendencias en puestos de liderazgo. Y el grupo 3 (azul), concentra la distribución de género en rangos académicos, productividad académica e investigación. - Figura 4.

La - Figura 4a hace referencia a las tendencias de los últimos años en investigación acerca de liderazgo femenino en medicina, los datos en amarillo son los que se incluyeron desde de 2016: diversidad, inclusión y equidad de género.

\section{Discusión}

En los últimos años, el liderazgo ha venido transformándose en diferentes áreas, donde la inclusión y la aceptación de la diversidad son objetivos claros a alcanzar. En línea con esa tendencia, se ha generado crecimiento y participación de la mujer como líder en el campo científico, ganando espacios de cambio dentro de la cultura organizacional. Sin embargo, aún existen barreras para alcanzar esos objetivos constituyendo un desafío para el desarrollo y el entendimiento del liderazgo femenino. ${ }^{9}$

Lo anterior, ha promovido un incremento en la base literaria del liderazgo femenino, haciendo necesario una valoración de las tendencias de publicación, como el primer paso, para definir la importancia que ha cobrado ese tema y precisar los puntos a trabajar y desarrollar a nivel local. Ese es el primer análisis bibliométrico publicado hasta el momento acerca del liderazgo femenino en medicina. Si bien eso limita el análisis y la comparación de los resultados, permite resaltar tres aspectos importantes: el comportamiento del porcentaje de publicaciones, el tipo de revistas que publican y los países que más han publicado.

En relación con las tendencias de publicación, es posible determinar la importancia que ha cobrado el tema en las últimas dos décadas, puesto que el $88 \%$ de las publicaciones son a partir del año 1996. Ese cambio podría explicarse por el creciente interés en documentar las discrepancias entre una mayor población de mujeres estudiantes de medicina versus los bajos porcentajes de cargos de liderazgo, donde según varios reportes de la literatura, solo el $15 \%$ llegan a ser jefes de departamentos científicos, $21 \%$ son profesoras de rango alto en universidades y $16 \%$ decanas de facultades científicas, con porcentajes inferiores al $10 \%$ en posiciones de liderazgo en especialidades quirúrgicas. ${ }^{10-14}$ Otro factor que podría explicar la creciente divulgación científica del tema a las puertas del nuevo milenio, estaría dada por la mayor visibilidad de la problemática del papel de la mujer en la fuerza laboral, planteada en escenarios de importancia global, como la cuarta conferencia mundial sobre la mujer en Beijín en $1995^{15}$

El segundo punto a destacar, es el tipo de revistas que publican; a pesar del aumento de publicaciones del tema, ellas se han realizado principalmente en revistas científicas con bajo FI, lo que conlleva a una pobre difusión, visibilidad y accesibilidad de ese tipo de investigaciones a los profesionales de la salud. Otra de las limitaciones, es que los resultados a menudo informan sobre la cantidad de las publicaciones, pero no es exacta en evaluar su calidad. El FI podría dar una aproximación, pero él por sí mismo, tiene diferentes inconvenientes ya que los criterios que eligen los autores para publicar suelen ser diversos y no dependen exclusivamente del FI.

Finalmente, este estudio documentó que los países desarrollados continúan siendo líderes en la producción de artículos científicos; Estados Unidos ocupó el primer lugar en cuanto a publicaciones, seguido del Reino Unido y Canadá. Eso refleja la importancia del rol de la mujer y su liderazgo en países industrializados y el enfoque en la práctica médica estadounidense en comparación con otras regiones geográficas. En América Latina los estudios publicados son muy escasos, siendo México el país con mayor producción científica en ese tema, seguido de Brasil. Colombia no cuenta con publicaciones indexadas hasta la fecha, por lo que las regiones menos desarrolladas deben mejorar la producción científica para tomar acciones de cambio en el rol de las mujeres en medicina.

Es importante aclarar que la identificación del país de publicación en PubMed se realiza únicamente con la dirección del primer autor, por tal motivo, la cantidad de artículos resultantes de colaboraciones multinacionales no se pueden determinar. Eso se considera una limitación del estudio, debido a que no se puede calcular la producción en países en desarrollo, ubicados en Latinoamérica, África y Oriente Medio. Adicionalmente, las publicaciones en PubMed al ser 

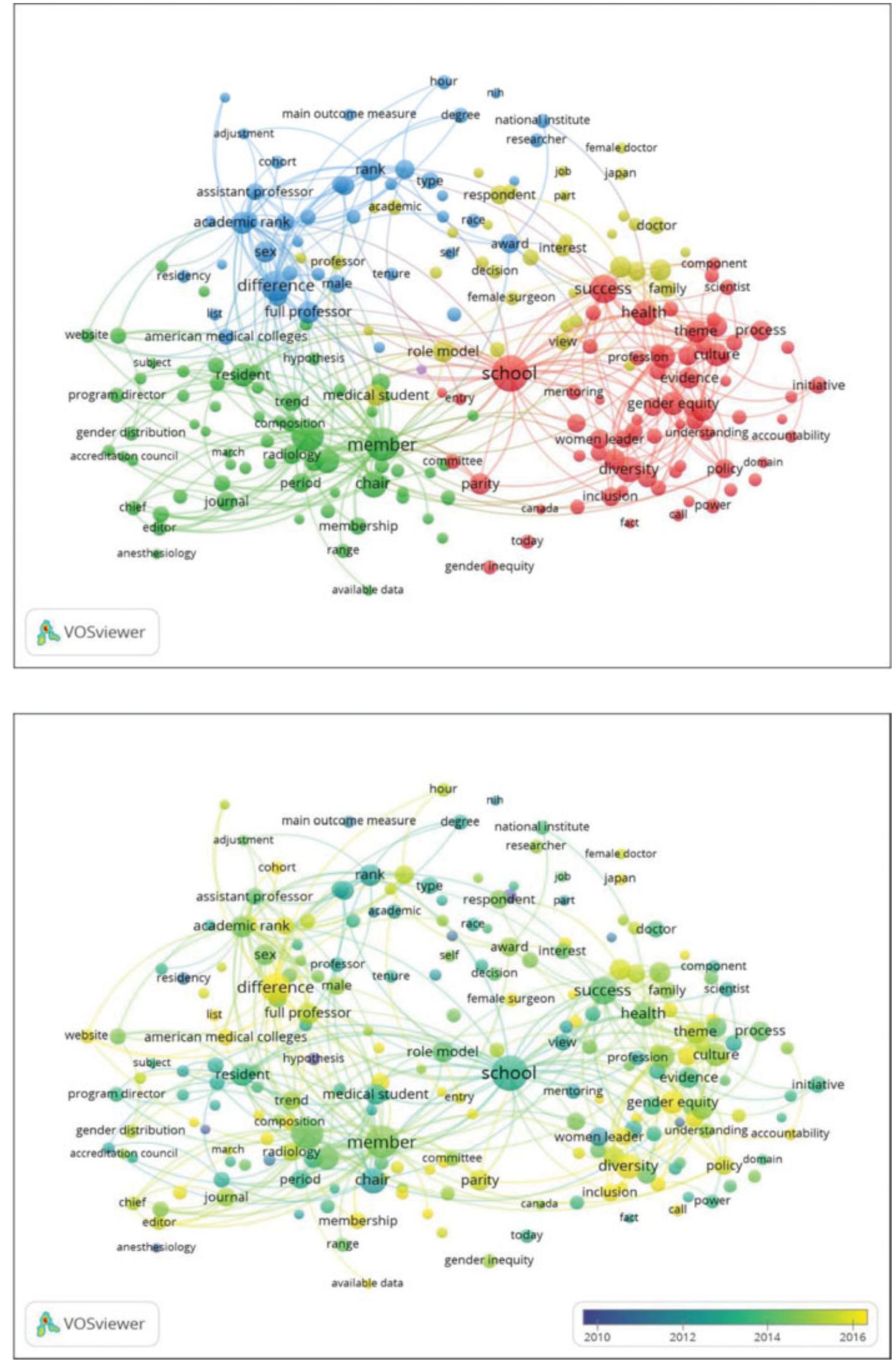

Fig. 4 Análisis y mapeo de co-ocurrencia en palabras clave-título y resumen. Análisis de co-ocurrencia en línea de tiempo. 
principalmente en inglés, pueden desfavorecer a países que publican en revistas en un idioma diferente.

Con el propósito de identificar, entender y conocer la actualidad en las publicaciones relacionadas con el liderazgo femenino y con el fin de visualizar las carencias $\mathrm{y} / \mathrm{o}$ debilidades en la actividad profesional y académica de las mujeres en medicina, se realizó un análisis por mapeo, donde se logró identificar tres grupos de importancia. El primero hace referencia a la participación en escuelas y la academia, el cual aumentó en las últimas décadas, el segundo está relacionado con las tendencias en liderazgo y el tercero con la distribución de género. Esos dos últimos grupos representan la mayor debilidad y es ahí donde debemos concentrar todos los esfuerzos para lograr un mayor impacto.

Las fortalezas principales del presente estudio incluyen una gran base literaria que abarcó varios años. Eso facilita la comprensión de las tendencias de publicación, lo que puede aplicarse no solo a urología, sino a la comunidad científica en general y abre la puerta al estudio del fenómeno en países latinoamericanos, donde la generación de trabajos de investigación ha sido muy escasa. A pesar de las limitaciones mencionadas, se considera que este estudio es un instrumento útil para investigadores.

\section{Conclusiones}

El liderazgo femenino en medicina y las publicaciones sobre el tema han aumentado de manera significativa durante las últimas dos décadas, principalmente en países industrializados. Latinoamérica debe unir sus esfuerzos en la producción de publicaciones en revistas de alto FI, para aumentar la visibilidad de la mujer en medicina, con el fin de tener una base real para trabajar por la equidad de género.

Conflicto de intereses

Los autores declaran no tener ningún conflicto de intereses.

\section{Referencias}

1 Buckley LM, Sanders K, Shih M, Kallar S, Hampton C. Obstacles to promotion? Values of women faculty about career success and recognition. Committee on the Status of Women and Minorities, Virginia Commonwealth University, Medical College of Virginia Campus. Acad Med 2000;75(03):283-288

2 Morahan PS, Rosen SE, Richman RC, Gleason KA. The leadership continuum: a framework for organizational and individual assessment relative to the advancement of women physicians and scientists. J Womens Health (Larchmt) 2011;20(03):387-396

3 Zhuge Y, Kaufman J, Simeone DM, Chen H, Velazquez OC. Is there still a glass ceiling for women in academic surgery? Ann Surg 2011;253(04):637-643

4 Reed V, Buddeberg-Fischer B. Career obstacles for women in medicine: an overview. Med Educ 2001;35(02):139-147

5 Bickel J, Wara D, Atkinson BF, et al; Association of American Medical Colleges Project Implementation Committee. Increasing women's leadership in academic medicine: report of the AAMC Project Implementation Committee. Acad Med 2002;77(10):1043-1061

6 Arbeláez Gómez M, Onrubia Goñi J. Análisis bibliométrico y de contenido. Dos metodologías complementarias para el análisis de la revista colombiana Educación y Cultura. Revista de Investigaciones UCM 2014;14(23):14-31

7 InCitesTM Journal Citation Reports. Journal data filtered by selected year JCR year: 2014 . Thomson Reuters 2015. SCIE, SSCI

8 Van Eck NJ, Waltman L, Dekker R, Van Den Berg J. A comparison of two techniques for bibliometric mapping: multidimensional scaling and VOS. J Am Soc Inf Sci Technol 2010;61:2405-2416. Doi: 10.1002/asi.21421

9 Waltman L, van Eck NJ, Noyons ECM. A unified approach to mapping and clustering of bibliometric networks. 2010http:// arxiv.org/abs/1006.1032. Accessed 13 Sep 2018

10 Alwazzan L, Al-Angari SS. Women's leadership in academic medicine: a systematic review of extent, condition and interventions. BMJ Open 2020;10(01):e032232. Doi: 10.1136/ bmjopen-2019-032232

11 Donnally CJ III. Trends in Leadership at Spine Surgery Fellowships. SPINE An International Journal for the study of the spine, Publish Ahead of Print DOI: . Doi: 10.1097/BRS.0000000000003350

12 Robinson M, Macneily A, Afshar K, et al. Leadership in Canadian urology: what is the right stuff? J Surg Educ 2013;70(05): 606-612. Doi: 10.1016/j.jsurg.2013.04.013

13 Konanur A, Egro FM, Kettering CE, et al. Gender Disparities Among Burn Surgery Leadership. J Burn Care Res 2020;41(03):674-680

14 Bibliometric Analysis of Authorship Trends and Collaboration Dynamics over the Past Three Decades of B_O_N_E_'s_ _Publication History Bone. 2018 February;107:27-35. doi:10.1016/j. bone. 2017.10.026

15 Beatrice E Avolio y Giovanna F. Di Laura, Progreso y evolución de la inserción de la mujer en actividades productivas y empresariales en América del Sur, Revista de la CEPAL N 122 • Agosto de 2017 